\title{
Influence of Sonographic Imaging on Patients with Anterior Abdominal Wall Hernias to Prevent Reoperations
}

\author{
Ali Enshaie ${ }^{1}$, Saeed Kashefi ${ }^{2}$, Vahideh Aghamohammadi ${ }^{3}$, Seyfollah Rezaie ${ }^{4}$, Niloofar Afshari ${ }^{5}$, Khadijeh Nasiri ${ }^{6}$
}

\begin{abstract}
Background: Hernia is defined as an area of weakness or complete disruption of the body wall's fibromuscular tissues. Structures arising from the cavity contained by the body wall can pass through, or herniate, through such a defect. The typical clinical finding is a bulged mass increasing in size when intra-abdominal pressure rises. The hernia is asymptomatic or may cause severe pain for patients. Arising of intra-abdominal pressure for each reason can generate anterior abdominal wall hernias; on the contrary, each synchronous surgically treatable intra-abdominal disease can be revealed with the same symptoms, and distinction of this disease prior to the surgery is important.

Materials and methods: This study was conducted on 90 patients who were candidates for anterior abdominal wall herniorrhaphy. All patients were screened for the coexistence of intra-abdominal surgically treatable diseases using the abdominopelvic sonographic examination. According to our project, patients with a synchronous intra-abdominal illness were treated with single surgery for their hernia and surgically treatable disease. Other patients with the healthy sonographic report were only subject to herniorrhaphy.

Results: The sonographic report was normal in 53 patients and abnormal (including cholelithiasis or any synchronous surgically treatable disease) in 37 patients. The study of the population using the Chi-square test to determine the need for further surgery (normal sonographic report rate) showed a statistical difference between hernia groups $(p=0.001)$. In the umbilical hernia group, the need for further surgery is significantly lower than that in the other groups $(p<0.001)$.

Conclusions: The coexistence of intra-abdominal surgically treatable disease with anterior abdominal wall hernias and their possible recurrence due to the remaining of the intra-abdominal illness as a source for intra-abdominal cavity pressure convinced surgeons to carefully check patients for each surgically treatable intra-abdominal disease before surgery.

Keywords: Abdominal wall hernia, Cholelithiasis, Sonography.

World Journal of Laparoscopic Surgery (2021): 10.5005/jp-journals-10033-1445
\end{abstract}

\section{INTRODUCTION}

Anterior abdominal wall hernias are described as the weakness in the fibromuscular layer of the abdominal wall, which can be congenital or acquired. Based on their arising anatomic region, hernias are divided into umbilical, epigastric, primary ventral, secondary ventral (incisional), and Spigelian subtypes. An umbilical hernia is the most common type of hernia and is generally prevalent in premature newborns. The incidence of umbilical hernia in the adult is mostly unknown, but most cases are thought to be acquired rather than congenital. It is known to occur more commonly in adult females (with a 3:1 ratio). An umbilical hernia is more commonly found in association with processes that increase intra-abdominal pressure. ${ }^{1}$ Almost all surgeons prefer conservative treatment for umbilical hernia in children up to 5 years old. ${ }^{2}$ Epigastric hernias occurring between umbilicus and xiphoid processes are prevalently detected in older adults versus women with a 3:1 ratio. Secondary ventral hernias prevalently occur after abdominal incisions, and small incisions can prevent such hernias. Risk factors for these hernias include increasing age, malnutrition, ascites, diabetes, obesity, smoking, long-term corticosteroid consumption, sepsis after surgery, wound infections, and emergency surgeries. Spigelian hernia is present near the arcuate line exactly lateral to rectus abdominis muscle. The diagnosis of Spigelian hernia is associated with challenges because of its complex regional anatomy. ${ }^{1}$ European Hernia Association has presented a general classification of primary anterior abdominal wall hernias, the consideration of which is helpful to conduct the study. Based on this classification, anterior abdominal hernias are at midline or

\begin{abstract}
1,2,4 Department of General Surgery, School of Medicine, Urmia University of Medical Sciences, Urmia, Iran

${ }^{3}$ Department of Nutrition, Khalkhal University of Medical Sciences, Khalkhal, Iran

${ }^{5}$ Department of Dermatology, School of Medicine, Tabriz University of Medical Sciences, Tabriz, Iran

${ }^{6}$ Department of Nursing, Khalkhal University of Medical Sciences, Khalkhal, Iran

Corresponding Author: Saeed Kashefi, Department of General Surgery, School of Medicine, Urmia University of Medical Sciences, Urmia, Iran, Phone: +984431988000, +989143478332, e-mail: s.kashefi.s 1986@gmail.com

How to cite this article: Enshaie A, Kashefi S, Aghamohammadi V, et al. Influence of Sonographic Imaging on Patients with Anterior Abdominal Wall Hernias to Prevent Reoperations. World J Lap Surg 2021;14(2):111-113.
\end{abstract}

Source of support: Nil

Conflict of interest: None

lateral of the abdomen, namely medial to oblique and lateral to the lateral border of rectus abdominis muscle, respectively. In terms of size, they are small $(<2 \mathrm{~cm})$, midsize $(2-4 \mathrm{~cm})$, or large $(>4 \mathrm{~cm}){ }^{3}$ Diagnosis of all hernia types is made clinically, and they can be treated by laparoscopic or classic methods. ${ }^{1}$ Another aspect of this study was related to synchronous intra-abdominal surgically treatable diseases, the most prevalent of which is cholelithiasis. 
Gallstone formation is a widespread disease in the gastrointestinal system, and the following factors can increase its incidence rate: Increasing age, female gender, obesity, pregnancy, familial heredity and nutritional habits, Crohn, spherocytosis, sickle cell anemia, thalassemia, and patients experiencing gastric or ileal resections. ${ }^{2}$ Diagnostic sonography is the primary modality to detect gallstones. Pathophysiology of anterior abdominal wall hernias can be related to two series of factors. The first is related to each problem that elevates intra-abdominal pressure, including obesity, pregnancy, ascites, bowel obstruction, and peritoneal dialysis. The second is associated with structural and functional weakness of muscles, tendons, and fascia of the abdominal wall. ${ }^{1}$ Congenital or acquired impairment in the metabolism of the collagen production cycle (for example, due to smoking or malnutrition) plays an essential role in creating the disease. The deficiency in the collagen production cycle causes two classes of disorders: Molecular-cellular and extracellular matrix diseases. Extracellular matrix disease is the primary pathophysiology of ventral hernias. ${ }^{4}$ Having common risk factors for anterior abdominal hernias and cholelithiasis (as the most common synchronous surgically treatable disease) and its synchronous existence probability if not considered, it would be harmful to patients and healthcare systems. ${ }^{5,6}$ Since the demonstration of the simultaneous existence of intra-abdominal surgically treatable diseases has two main advantages for patients, first, the time to diagnose and evaluate the synchronous condition and second, saving laparoscopy as a surgical option for the treatment of patients. This approach to anterior abdominal wall hernias has a lot of financial benefits for healthcare systems.

\section{Materials and Methods}

Ninety patients referred to the general surgery clinic of Urmia Imam Khomeini Hospital for anterior abdominal wall herniorrhaphy were studied from September 2017 to September 2018. Our inclusion criteria were the patients who had anterior abdominal hernias. This trial, approved by the Medical Ethics Committee of the Urmia University of Medical Sciences, Urmia, Iran, is in accordance with the Declaration of Helsinki (approval number: IR. UMSU. REC. 97.1857). Subjects meeting the inclusion criteria were recruited in this study after obtaining their written consent. Exclusion criteria were incarcerated, or strangulated hernias, pregnant women, patients with a history of psychiatric drug consumption, and patients with end-stage cardiopulmonary disease or chronic renal failure, and none of the patients had a history of opium addiction. In this step, all patients screened with the abdominopelvic sonographic examination. The patients were divided into four groups: Umbilical, epigastric, ventral, and Spigelian hernias; and two sub-types: Group 1 (with normal sonographic report) treated only with herniorrhaphy and group 2 (including cholelithiasis or any synchronous surgically treatable disease) who were subject to a single surgery for treating both disorders. All patients were screened with a complete abdominopelvic sonographic examination.

\section{Statistical Analysis}

All statistical analyses were fulfilled using IBM SPSS Statistics software (version 24) (IBM SPSS Statistics, Armonk, United States). A $p$-value of less than 0.05 was regarded to be statistically significant. A Chi-square test was used for categorical data to identify significant differences. The comparisons of the age difference between the hernia types were performed with the analysis of variance (ANOVA).

\section{Results}

Ninety patients admitted for anterior abdominal wall herniorrhaphy from September 2017 to September 2018 were studied. The mean average age of the population was $49.17 \pm 12.17$ years. The intraabdominal surgically treatable disease was reported in 37 patients, and the report was normal in 53 patients. The characteristics of the subjects in the hernia groups are shown in Table 1. The age means of participants between the hernia groups using one-way ANOVA showed no statistical significance $(p=0.524)$, which was confirmed by the post hoc test $(p>0.05)$. Concerning gender ratio in the population, a majority of patients were women ( $82.2 \%$ women vs $17.8 \%$ men), and hernia groups had significant difference regarding sex ratio $(p=0.019)$. Sonographic reports of patients were interpreted as follows. In the umbilical hernia group, eight cases of cholelithiasis and six cases of an abdominal mass (including two cases of uterine myoma, two cases of ovarian cancer, a case of HCC, and a case of sigmoid cancer) were reported. In the epigastric hernia group, four cases of cholelithiasis and three cases of an abdominal mass (including two cases of simple ovarian cysts and a gastric gastrointestinal stromal tumor) were observed (Table 2). Two cases of cholelithiasis and six cases of an abdominal mass (including two cases of the myomatous uterus, two cases of large uterine myoma, and two cases of advanced uterine cancer) were reported in the ventral hernia group. All reports of the Spigelian hernia group were normal. In other words, there were 14 cases from 58 umbilical hernia patients, 7 cases from 20 epigastric hernia patients, and 8 cases from 10 ventral hernia patients, requiring a single surgery for their concomitant hernia and intra-abdominal disease. The study of the population using the Chi-square test to determine the need for further surgery (normal sonographic report rate) showed a statistical difference between the hernia groups $(p=0.001)$. In the umbilical hernia group, the need for further surgery is significantly lower than that in the other groups $(p<0.001)$ (Table 2$)$

\section{Discussion}

Anterior abdominal wall hernias are congenital or acquired and are divided into umbilical, epigastric, ventral (incisional), and Spigelian hernias according to their anatomic region. An umbilical hernia is the most common type that is generally prevalent in premature newborns, and familial heredity has a recognized role in the incidence of this disease. Umbilical hernias in adolescents are acquired and more commonplace in women than in men (with a 3:1 ratio). Although our study confirmed the higher incidence of umbilical hernia (64.4\%) among

Table 1: Characteristics of the subjects in the hernia groups

\begin{tabular}{|c|c|c|c|c|c|}
\hline Hernia groups $(N)$ & Age $($ Mean $\pm S D)$ & $p_{1}$ & $\operatorname{Men} N(\%)$ & Women N (\%) & $p_{2}$ \\
\hline Umbilical hernia group $(n=58)$ & $11.36 \pm 47.74$ & \multirow{4}{*}{0.524} & $8(13.79)$ & $50(86.2)$ & \multirow{4}{*}{0.019} \\
\hline Epigastric hernia group $(n=20)$ & $13.53 \pm 51.80$ & & $8(40)$ & $12(60)$ & \\
\hline Ventral hernia group $(n=10)$ & $14.92 \pm 51.60$ & & $2(20)$ & $8(80)$ & \\
\hline Spigelian hernia group $(n=2)$ & $3.53 \pm 52.50$ & & $0(0)$ & $2(100)$ & \\
\hline
\end{tabular}

$p_{1}$, using one-way ANOVA test; $p_{2}$, using Chi-square test 
Table 2: Types of hernia description and sonographic report among study population

\begin{tabular}{|c|c|c|c|c|c|c|}
\hline \multirow{4}{*}{$\begin{array}{l}\text { Hernia types } \\
\text { Umbilical hernia }\end{array}$} & \multirow{2}{*}{\multicolumn{2}{|c|}{ Frequency }} & \multicolumn{3}{|c|}{ Sonographic report } & \multirow{4}{*}{$\frac{p}{>0.001}$} \\
\hline & & & \multirow{2}{*}{$\frac{\text { Normal }}{44}$} & \multirow{2}{*}{$\begin{array}{c}\text { Cholelithiasis } \\
8\end{array}$} & \multirow{2}{*}{$\frac{\text { Abdominal mass }}{6}$} & \\
\hline & $N$ & 58 & & & & \\
\hline & $\%$ & 64.4 & 75.8 & 13.8 & 10.34 & \\
\hline \multirow[t]{2}{*}{ Epigastric hernia } & $N$ & 20 & 13 & 4 & 3 & \multirow{2}{*}{0.035} \\
\hline & $\%$ & 22.2 & 65 & 20 & 15 & \\
\hline \multirow[t]{2}{*}{ Ventral hernia } & $N$ & 10 & 2 & 2 & 6 & \multirow{2}{*}{0.202} \\
\hline & $\%$ & 11.1 & 20 & 20 & 60 & \\
\hline \multirow[t]{2}{*}{ Spigelian hernia } & $N$ & 2 & 2 & - & - & \multirow{2}{*}{ - } \\
\hline & $\%$ & 2.2 & 100 & - & - & \\
\hline
\end{tabular}

$p$, using Chi-square test

all the patients, the results about gender prevalence showed higher prevalence in women compared to the previous studies (with a 6:1 ratio). Epigastric hernias are prevalently detected in elderly men than in women with a 3:1 ratio; however, our research showed different results regarding epigastric hernia. In our population, the epigastric hernia was 1.5 times more prevalent in women. Ventral hernias occur after abdominal incisions, and small incisions are preventing factors of this hernia type. Risk factors for a ventral hernia include age, malnutrition, ascites, diabetes, obesity, smoking, long-term corticosteroid use, sepsis after surgery, wound infections, and emergency surgeries. ${ }^{1}$

Nevertheless, our study showed different results; if technical mistakes or the risks as mentioned above are absent, there is an $80 \%$ association between ventral hernias and intra-abdominal surgically treatable diseases. Spigelian hernia is observed near the arcuate line exactly lateral to rectus abdominis muscle. Because of its complex regional anatomy, the diagnosis of Spigelian hernia is accompanied by challenges. In our study, only $2.2 \%$ of the population had Spigelian hernia, confirming the challenging diagnosis of this type of hernia according to texts. Another aspect of our research was related to the coexistence of intra-abdominal surgically treatable diseases. As a prevalent intra-abdominal disease, cholelithiasis has common risk factors with anterior abdominal wall hernias. There are two main pathophysiologic factors for anterior abdominal wall hernias: First, any factor elevating intra-abdominal pressure such as obesity, pregnancy, ascites, bowel obstruction, and intra-peritoneal dialysis; and second, structural and functional weakness of anterior abdominal wall muscles, tendons, and fascia. ${ }^{1}$ This statement has been confirmed by Christian Nordqvist's article published in Medical News Today magazine in 2016, which demonstrated each cause of elevated intra-abdominal pressure, including multiple pregnancies and frequent coughs as the primary pathophysiology of umbilical hernias. ${ }^{5}$ Among other researches in this context, the retrospective Briant et al. research conducted from 1962 to 1967 in the Kentucky University Hospital in the USA on 66 women referred to surgery clinic with an umbilical hernia can be mentioned, which confirmed cholelithiasis coexistence in $40 \%$ of patients. ${ }^{6}$ The two studies, as discussed above, were similar to ours, in which $24.4 \%$ of patients with umbilical hernia had a concomitant intra-abdominal surgically treatable disease. Another research by Chen et al. ${ }^{7}$ was conducted on 7,570 patients in China, in which 918 cases referred for routine checkups had cholelithiasis. In this study, they proved that patients with metabolic syndrome are at a five times higher risk of cholelithiasis than other patients. ${ }^{7}$ In another study by Kaymak et al. conducted on 2015 in Turkey with 78 patients admitted for laparoscopic cholecystectomy, 11 patients (14\%) had an umbilical hernia, 39 patients (50\%) had metabolic syndrome, and eight patients (10.2\%) had both umbilical hernia and metabolic syndrome. This study again emphasized the role of metabolic syndrome as a risk factor for gallstone formation. At the same time, the study results showed that the prevalence of umbilical hernia is higher in cholelithiasis patients. The last two studies predicted the common risk factors of umbilical hernia and cholelithiasis, and our results were similar to them so that there was the coexistence of cholelithiasis among $13.8 \%$ of umbilical hernia patients..$^{8,9}$

\section{Conclusion}

Emphasizing the results of our study, the probable coexistence of surgically treatable intra-abdominal disease with the same symptoms is not negligible in the patients with anterior abdominal wall hernias. Therefore, it is recommended to perform total abdominopelvic sonographic examination on patients with these hernias before their herniorrhaphies.

\section{ACKnOWledgments}

All members of the surgery department in the Urmia University of Medical Sciences were cordially available to answer our questions. We would also like to thank the experts from other universities who were involved in the validation of the survey for this research.

\section{References}

1. Zinner MJ. Maingot's abdominal operations. McGraw-Hill, Health Pub Division; 2015.

2. Brunicardi F, Andersen D, Billiar T, Dunn D, Hunter J, Matthews J, Pollock R. Schwartz's principles of surgery, 10e. McGraw-hill; 2014.

3. Petro CC, Novitsky YW. Classification of hernias. Springer International Publishing Switzerland; 2016.

4. Michael FG. The biology of hernia formation. Surg Clin North Am 2008;88(1):1-15, vii. DOI: 10.1016/j.suc.2007.10.007.

5. Nordqvist Ch. Umbilical hernia: what you need to know. Medical News Today 2019:1-1.

6. Bryant M, Griffen W. Umbilical hernia and gallbladder disease. Am J Surg 1969;117(5):653-655. DOI: 10.1016/0002-9610(69)90400-0.

7. Chen LY, Qiao QH, Zhang SC, et al. Metabolic syndrome and gallstone disease. World J Gastroenterol 2012;18(31):4215-4220. DOI: 10.3748/ wjg.v18.i31.4215.

8. Kaymak Ş, Lapsekili E, Demirbaş S. The effect of obesity on co-existence of umbilical hernia and metabolic syndrome in patients with cholelithiasis. JCAM 2015;1-4. DOI: 10.4328/JCAM.3232.

9. Yamanaka T, Miyazaki T, Kumakura Y, et al. Umbilical hernia with cholelithiasis and hiatal hernia: a clinical entity similar to Saint's triad. J Surg Case Rep 2015. DOI: 10.1186/s40792-015-0067-8. 